\title{
BMJ Open Effect of perioperative intravenous lidocaine on postoperative outcomes in patients undergoing resection of colorectal cancer: a protocol for systematic review and meta-analysis
}

Wentao Jl (D) , Xiaoting Zhang, Guolin Sun, Xiandong Wang, Jia Liu, Jinjun Bian, Lulong Bo

To cite: JI W, Zhang X, Sun G, et al. Effect of perioperative intravenous lidocaine on postoperative outcomes in patients undergoing resection of colorectal cancer: a protocol for systematic review and meta-analysis. BMJ Open 2021;11:e048803. doi:10.1136/ bmjopen-2021-048803

- Prepublication history and additional supplemental material for this paper are available online. To view these files, please visit the journal online (http://dx.doi.org/10.1136/ bmjopen-2021-048803).

WJ, XZ and GS contributed equally.

Received 11 January 2021 Accepted 09 August 2021

Check for updates

(c) Author(s) (or their employer(s)) 2021. Re-use permitted under CC BY-NC. No commercial re-use. See rights and permissions. Published by BMJ.

Faculty of Anesthesiology, Changhai Hospital, Shanghai, China

\section{Correspondence to}

Dr Lulong Bo;

bartbo@smmu.edu.cn and

Dr Jinjun Bian;

jinjunbicu@163.com

\section{ABSTRACT}

Introduction Techniques using local anaesthetics provide high-quality analgesia, while the anti-inflammatory properties of these drugs may represent an additional advantage. Perioperative intravenous lidocaine has shown positive effects not only on postoperative pain but also on bowel function and duration of hospital stay, due to its analgesic, anti-inflammatory and opioid-sparing effects. However, these potential benefits are not well established in patients undergoing resection with colorectal cancer. This research aims to determine the effect of perioperative intravenous lidocaine on postoperative outcomes in patients undergoing resection of colorectal cancer.

Methods and analysis PubMed, Embase, Web of Science, CNKI, SinoMed and WanFang Data databases were electronically retrieved to include the randomised controlled trials comparing perioperative intravenous lidocaine with placebo infusion in patients undergoing resection of colorectal cancer before August 2021. Registers of clinical trials, potential grey literature and abstracts from conferences will also be searched. Two reviewers will screen literature, extract data and assess risk of bias of studies included independently. The primary outcome variable will be long-term survival outcome, tumour recurrence and metastasis rate, and restoration of intestinal function. The secondary outcome variables will consist of the severity of postoperative pain at 4, 12, 24 and 48 hours after surgery, the incidence of postoperative nausea and vomiting, and the length of hospital stay. A meta-analysis will be performed using RevMan V.5.4 software provided by the Cochrane Collaboration and Stata V.12.0. subgroup and sensitivity analyses will be conducted.

Ethics and dissemination Because the data used for this systematic review will be exclusively extracted from published studies, ethical approval and informed consent of patients will not be required. The systematic review will be published in a peer-reviewed journal, presented at conferences and shared on social media platforms.

PROSPERO registration number CRD42020216232.

\section{Strengths and limitations of this study}

- This research will provide the best assessment with currently available data on whether perioperative intravenous lidocaine can improve postoperative outcomes in patients undergoing resection of colorectal cancer.

- The analysis of various sources of heterogeneity and the assessment of risk of bias of the included studies will be a critical point for extracting and synthesising evidence-based conclusions.

- One limitation of this study is that differences in duration of perioperative intravenous lidocaine as interventions cannot be restricted, which might affect results of this study.

- Notably, this research will include only patients with colorectal cancer, which differs from other metaanalyses and may be an advantage or a challenge.

\section{INTRODUCTION}

Perioperative intravenous lidocaine (IVL) infusion showed potential advantages in a range of surgical specialties, including hepatobiliary, ${ }^{1}$ gynaecological and colorectal surgery. ${ }^{2}$ Local anaesthetics may have some effects on cancer cell viability and migration. ${ }^{45}$ Several preclinical studies have shown that lidocaine has a prominent antitumour activity on multiple cancer cells and is a promising therapeutic agent for the treatment of cancer. ${ }^{6-8}$ However, the effect of IVL on the postoperative outcomes of patients with colorectal cancer is controversial. Studies have suggested that IVL conveys postoperative benefits including reduction of postoperative pain, and shortened time to return of gastrointestinal function. ${ }^{9-12}$ However, a recent randomised, double-blinded, placebocontrolled trial by Herzog et al indicated that IVL had no significant benefits for 
patients undergoing robot-assisted colorectal surgery, ${ }^{13}$ including cumulated morphine consumption at 24 hours or 72 hours after end of surgery, considering multiple outcomes including time until first flatus or defecation, use of antiemetics and time until discharge.

We hypothesised that for patients with colorectal cancer, perioperative lidocaine given intravenously would have benefits on long-term survival outcome, reduce or delay the chance of tumour recurrence or metastasis, improve the restoration of intestinal function, relieve pain, reduce the incidence of postoperative nausea and vomiting (PONV) and shorten the length of hospital stay (LOS) after surgery.

\section{METHODS}

This protocol has been registered on the PROSPERO based on the Preferred Reporting Items for Systematic Review and Meta-Analysis Protocol (PRISMA-P) guidelines. The protocol will follow the Meta-analysis of Observational Studies in Epidemiology ${ }^{14}$ the Cochrane Handbook for Systematic Reviews of Interventions and the PRISMA-P statement guideline. ${ }^{15}$

\section{Inclusion criteria for study selection \\ Types of studies}

All studies designed as randomised controlled trials (RCTs) will be included. The current clinical trial results will be objectively integrated, which is conducive to the evaluation of the efficacy of IVL on postoperative outcomes in patients undergoing resection of colorectal cancer. Exclusion criteria comprised paediatric patients, non-colorectal or emergency procedures, non-RCT methodology and lack of any relevant clinical outcome measures. We will also exclude reviews, qualitative studies, animal trials and laboratory studies. Studies that included more than two study arms, but had IVL and placebo groups, were included and only those groups pertinent to this meta-analysis were considered.

\section{Types of patients}

Patients scheduled for resection of colorectal cancer will be included in this study. Other restrictions included age ( $\geq 18$ years old) and American Society of Anesthesiologists' (ASA) physical status (I-III).

\section{Types of interventions}

Perioperative IVL is administrated as the intervention. Normal saline as placebo or no intervention could be administrated in the control groups. No consideration was given to how long the lidocaine infusion was continued after surgery, but to be eligible for inclusion, the infusion had to commence before the surgical incision.

\section{Types of outcome measures}

\section{Primary outcomes}

The primary outcome variables are long-term survival outcome as reported and defined by the original studies, the occurence of tumour recurrence or metastasis and the restoration of intestinal function, including the time until first postoperative flatus and defecation. Flatus and defecation are important indications for exclusion of intestinal obstruction and restoration of intestinal function postoperatively. ${ }^{16}$

\section{Secondary outcomes}

The secondary outcome variables include the severity of pain measured using Visual Analogue Score (VAS) on postoperative days at 4, 12, 24 and 48 hours after surgery, the incidence of PONV and the LOS.

\section{Search methods for the identification of studies Electronic searches}

Six electric databases (PubMed, Embase, Web of Science, CNKI, SinoMed and WanFang Data) will be searched without language restriction to identify RCTs published before August 2021. A search strategy has been developed for the six databases as a combination of "Colonic Neoplasms", "Rectal Neoplasms" or "Colorectal Neoplasms" in all fields and "lidocaine" or "lignocaine" in all fields and "Infusions" or "Intravenous" in all fields and "Randomized Controlled Trial" or "RCT" in all fields. The reference lists will be searched manually for potentially relevant articles.

The search strategy for PubMed is described in online supplemental table 1 , which will include all search terms, and other searches will be carried out based on those results. This will be suitably adapted to search in the other databases. There are no limits on language and publication status.

\section{Searching other resources}

We will also search PROSPERO, the International Clinical Trials Registry Platform, ClinicalTrials. gov, dissertations and grey literature to identify systematic reviews or clinical trials related to IVL. Manual searches will be conducted for related journals and conference processes.

\section{Data collection and analysis \\ Selection of studies}

Two reviewers (JW and ZX) will screen the search results according to the title and abstract independently. After the full text is obtained, the two reviewers will screen the references for potentially relevant studies. Any discord will be resolved by discussion between the two authors and an arbiter (SG). The selection procedure for the study will be summarised and shown in a PRISMA flow chart (figure 1).

\section{Data extraction}

Based on the inclusion criteria, a standard form of data collection will be produced prior to data extraction. The two reviewers (JW and ZX) will independently extract data on patient baseline demographics (age, sex, ASA physical status), operative variables and details of the lidocaine administration (dose, starting point, perioperative duration and any bolus dose administered) as well as the placebo. The studies included were stratified according 


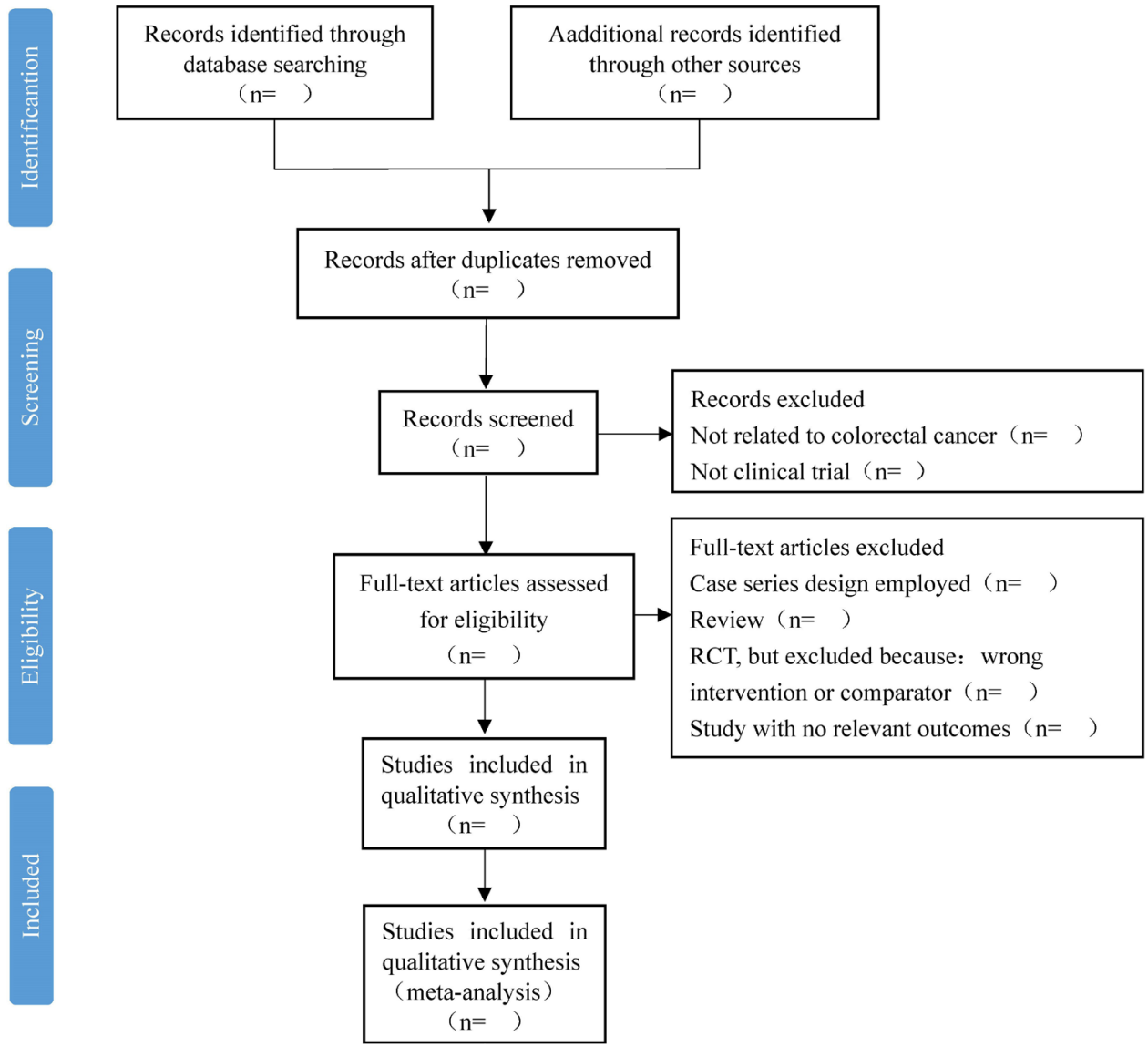

Figure 1 The PRISMA flow chart of the selection process. PRISMA, Preferred Reporting Items for Systematic Reviews and Meta-Analyses; RCT, randomised controlled trial.

to whether the patients underwent open or laparoscopic resection. If the data necessary for meta-analysis of continuous variables were not available, the corresponding author was approached to provide the raw data, and if a response was not received, the technique described by Hozo et al was employed to estimate the mean and SD from the median and IQR. ${ }^{17}$ When the consensus on data extraction is not available through discussion, the third reviewer $(\mathrm{SG})$ will make a decision.

\section{Assessment of study quality}

The Cochrane Collaborations tool will be used to assess selection bias, performance bias, attrition bias and reporting bias. Two reviewers (JW and ZX) will independently rate the quality of the RCTs and fulfill the items of risk of bias as low, high or unclear. Any discrepancies between the two reviewers will be solved by a consulting group including two experts (WX and SG). The quality of evidence resulting from this systematic review was evaluated through the Grading of Recommendations Assessment, Development and Evaluation, and the level of evidence will be classified as high, medium, low or very low. ${ }^{18}$

\section{Statistical analyses and data synthesis}

RevMan V.5.4 will be used for data synthesis. The pooled effects of dichotomous outcomes will be analysed as risk ratio using the Mantel-Haenszel technique and 95\% CIs. The pooled effects of continuous outcomes will be analysed using mean difference and $95 \%$ CI. A p value of less than 0.05 will be considered to be statistically significant.

\section{Assessment of heterogeneity}

$\mathrm{I}^{2}$ statistic will be used to estimate statistical heterogeneity ( $\mathrm{I} \leq 50 \%$ as low heterogeneity, $\mathrm{I}^{2}>50 \%$ as high heterogeneity). Clinical heterogeneity will be assessed by the two reviewers (JW and ZX) and the consulting group (WX and SG). If high clinical or statistical heterogeneity is observed, a random effect model will be used. Otherwise, a fixed effect model will be chosen.

\section{Assessment of publication bias}

A funnel plot will be used to assess publication bias when 10 or more RCTs are available for quantitative analysis. Egger test will be performed if included studies are less than $10 .{ }^{19}$ For Egger's test, $\mathrm{p}$ value of greater than 0.05 was determined as no significant publishing bias or smallstudy effects in studies. As funnel plot asymmetry does not necessarily suggest reporting bias, we will attempt to recognise potential causes for the asymmetry, including poor methodological quality and true heterogeneity of studies. 
Subgroup and sensitivity analyses

On detection of heterogeneity, a subgroup analysis will be carried out to judge the source of heterogeneity. The criteria for a subgroup analysis potentially include age, type of surgery, intervention dosage, frequency and duration. Considering the significant difference in the degree of trauma between laparoscopic surgery and open surgery, a subgroup analysis of surgical methods is necessary. ${ }^{20}$

Sensitivity analysis will be performed to determine the robustness of aggregate estimates and to detect whether any single study accounts for a substantial proportion of heterogeneity by eliminating the included studies from the summary review one by one. If low-quality articles are deleted, then a second meta-analysis will be carried out. Comparison and discussion of the results and effect size of the two meta-analyses will be held. ${ }^{21}$

\section{Trial sequential analysis}

Assessment of the risk of random errors will be done by trial sequential analysis (TSA). The results of TSA will determine whether the evidence in our meta-analysis is reliable and conclusive by providing the boundaries of sample size. $^{22}$

\section{Patient and public involvement}

Patients and the public will not participate in the study. However, once scientific publications disseminate our findings, they are circulated across social networks so that our conclusions will potentially affect the actions of anesthesiologists and health policymakers.

\section{Ethics and dissemination}

Because the data used for this systematic review will be exclusively extracted from published studies, ethical approval and informed consent of patients will not be required. The systematic review will be published in a peer-reviewed journal, presented at conferences and shared on social media platforms.

\section{DISCUSSION}

There has been increasing interest and evidence in the potential for IVL infusion in patients undergoing colorectal surgery. Greenwood et $a l^{23}$ considered that there is a wide safe range of plasma concentrations by monitoring the plasma concentration of lidocaine at different time points, which provides some evidence of the safety of continuous intravenous infusion of lidocaine.

A Cochrane review analysed a total of 68 RCTs across various surgical specialties and demonstrated an unclear effect of IVL versus placebo on pain scores, recovery of gastrointestinal function, postoperative nausea and overall opioid requirement. ${ }^{24}$

Specific to the field of colorectal surgery, a recently published meta-analysis within colorectal surgery provides support for the administration of perioperative IVL in terms of earlier return of gastrointestinal function, lower postoperative pain scores and reduced hospital LOS, ${ }^{25}$ with no difference in complication rates or apparent issues surrounding local anaesthetic toxicity. Another systematic review examined the role of IVL in the setting of elective colorectal surgery and concluded that IVL provided limited benefit in the reduction of early postoperative pain and morphine requirement when compared with placebo. ${ }^{26}$ Thus, a comprehensive systematic review and meta-analysis including new trials were warranted.

The underlying mechanisms of IVL might be multifactorial. Lidocaine has been shown to have anti-inflammatory, analgesic and opioid-sparing properties ${ }^{2728}$ which can improve the restoration of intestinal function, relieve pain, reduce the incidence of PONV and shorten the LOS in patients undergoing resection of colorectal cancer. Furthermore, lidocaine has a prominent antigrowth and antimetastatic effects on multiple cancer cells. ${ }^{78}$ Thus, IVL may have the potential to suppress the tumour recurrence or metastasis and improve the suvival rate of patients with colorectal cancer.

However, previous meta-analyses showed very different results. Meanwhile, systemic reviews or meta-analyses focused on the patients with colorectal cancer and antitumour effect of lidocaine seems to be absent. With the updated RCTs, the results of this meta-analysis will provide the most timely and comprehensive evidence on the efficacy of IVL in patients undergoing resection of colorectal cancer.

Acknowledgements We would like to thank the primary authors and sponsors that have provided access to their trial's database.

Contributors LB and JW designed the study. LB and BJ were the principal investigator and guarantor. JW, ZX and SG were the main coordinators of the study. JW, ZX, SG, WX and LJ conducted the study. SG and LB provided statistical and epidemiological support. JW wrote the article with the support of LB and ZX. All the authors revised and approved the final version of the manuscript.

Funding The authors acknowledged the support from 234 Climbing the Discipline Program of the first affilicated hospital of Naval Medical University (2020YXK053), and Shanghai Science and Technology Committee Rising-Star Program (19QA1408500).

Competing interests None declared.

Patient consent for publication Not required.

Provenance and peer review Not commissioned; externally peer reviewed.

Supplemental material This content has been supplied by the author(s). It has not been vetted by BMJ Publishing Group Limited (BMJ) and may not have been peer-reviewed. Any opinions or recommendations discussed are solely those of the author(s) and are not endorsed by BMJ. BMJ disclaims all liability and responsibility arising from any reliance placed on the content. Where the content includes any translated material, BMJ does not warrant the accuracy and reliability of the translations (including but not limited to local regulations, clinical guidelines, terminology, drug names and drug dosages), and is not responsible for any error and/or omissions arising from translation and adaptation or otherwise.

Open access This is an open access article distributed in accordance with the Creative Commons Attribution Non Commercial (CC BY-NC 4.0) license, which permits others to distribute, remix, adapt, build upon this work non-commercially, and license their derivative works on different terms, provided the original work is properly cited, appropriate credit is given, any changes made indicated, and the use is non-commercial. See: http://creativecommons.org/licenses/by-nc/4.0/.

ORCID iD

Wentao JI http://orcid.org/0000-0002-6602-6313 


\section{REFERENCES}

1 Zhao J-B, Li Y-L, Wang Y-M, et al. Intravenous lidocaine infusion for pain control after laparoscopic cholecystectomy: a meta-analysis of randomized controlled trials. Medicine 2018;97:e9771.

2 Samimi S, Taheri A, Davari Tanha F. Comparison between intraperitoneal and intravenous lidocaine for postoperative analgesia after elective abdominal hysterectomy, a double-blind placebo controlled study. J Family Reprod Health 2015;9:193-8.

3 Dewinter G, Coppens S, Van de Velde M, et al. Quadratus lumborum block versus perioperative intravenous lidocaine for postoperative pain control in patients undergoing laparoscopic colorectal surgery: a prospective, randomized, double-blind controlled clinical trial. Ann Surg 2018;268:769-75.

4 Liu H, Dilger JP, Lin J. Effects of local anesthetics on cancer cells. Pharmacol Ther 2020;212:107558.

5 Li R, Xiao C, Liu H, et al. Effects of local anesthetics on breast cancer cell viability and migration. BMC Cancer 2018;18:666.

6 Liu H, Dilger JP, Lin J. Lidocaine suppresses viability and migration of human breast cancer cells: TRPM7 as a target for some breast cancer cell lines. Cancers 2021;13 doi:10.3390/cancers13020234

7 Sun $\mathrm{H}$, Sun $\mathrm{Y}$. Lidocaine inhibits proliferation and metastasis of lung cancer cell via regulation of miR-539/EGFR axis. Artif Cells Nanomed Biotechnol 2019;47:2866-74.

8 Ye L, Zhang Y, Chen YJ, et al. Anti-tumor effects of lidocaine on human gastric cancer cells in vitro. Bratis/ Lek Listy 2019;120:212-7.

9 Dunn LK, Durieux ME. Perioperative use of intravenous lidocaine. Anesthesiology 2017;126:729-37.

10 Cooke C, Kennedy ED, Foo I, et al. Meta-analysis of the effect of perioperative intravenous lidocaine on return of gastrointestinal function after colorectal surgery. Tech Coloproctol 2019;23:15-24.

11 Tikuišis R, Miliauskas P, Samalavicius NE, et al. Intravenous lidocaine for post-operative pain relief after hand-assisted laparoscopic colon surgery: a randomized, placebo-controlled clinical trial. Tech Coloproctol 2014;18:373-80.

12 Paterson HM. Continuous intravenous lidocaine infusion for postoperative pain and recovery in adults. Tech Coloproctol 2019;23:69-71.

13 Herzog J, Schou M, Jensen KM. A randomised controlled trial of lidocaine infusion on post-operative opioid consumption in patients undergoing robotic colorectal surgery. Dan Med J 2020;67.

14 Stroup DF, Berlin JA, Morton SC, et al. Meta-analysis of observational studies in epidemiology: a proposal for reporting. meta-analysis of observational studies in epidemiology (MOOSE) group. JAMA 2000;283:2008-12.

15 Shamseer L, Moher D, Clarke M, et al. Preferred reporting items for systematic review and meta-analysis protocols (PRISMA-P) 2015 elaboration and explanation. BMJ 2015;350:g7647.

16 Buia A, Stockhausen F, Hanisch E. Laparoscopic surgery: a qualified systematic review. WJM 2015;5:238-54.

17 Hozo SP, Djulbegovic B, Hozo I. Estimating the mean and variance from the median, range, and the size of a sample. BMC Med Res Methodol 2005;5:13.

18 Guyatt GH, Oxman AD, Schünemann HJ, et al. GRADE guidelines: a new series of articles in the journal of clinical epidemiology. $J$ Clin Epidemiol 2011;64:380-2.

19 Egger M, Davey Smith G, Schneider M, et al. Bias in meta-analysis detected by a simple, graphical test. BMJ 1997;315:629-34.

20 Delaney CP, Marcello PW, Sonoda T, et al. Gastrointestinal recovery after laparoscopic colectomy: results of a prospective, observational, multicenter study. Surg Endosc 2010;24:653-61.

21 Han M, Nong L, Liu Z, et al. Safety and efficacy of mycophenolate mofetil in treating neuromyelitis optica spectrum disorders: a protocol for systematic review and meta-analysis. BMJ Open 2020;10:e040371.

22 Wetterslev J, Thorlund $\mathrm{K}$, Brok J, et al. Trial sequential analysis may establish when firm evidence is reached in cumulative meta-analysis. $J$ Clin Epidemiol 2008;61:64-75.

23 Greenwood E, Nimmo S, Paterson $\mathrm{H}$, et al. Intravenous lidocaine infusion as a component of multimodal analgesia for colorectal surgery-measurement of plasma levels. Perioper Med 2019;8

24 Weibel S, Jelting Y, Pace NL. Continuous intravenous perioperative lidocaine infusion for postoperative pain and recovery in adults. Cochrane Database Syst Rev 2018;6:CD009642.

25 Rollins KE, Javanmard-Emamghissi H, Scott MJ, et al. The impact of peri-operative intravenous lidocaine on postoperative outcome after elective colorectal surgery: a meta-analysis of randomised controlled trials. Eur J Anaesthesiol 2020;37:659-70.

26 MacFater WS, Rahiri J-L, Lauti M, et al. Intravenous lignocaine in colorectal surgery: a systematic review. ANZ J Surg 2017;87:879-85.

27 Cui W, Li Y, Li S, et al. Systemic administration of lidocaine reduces morphine requirements and postoperative pain of patients undergoing thoracic surgery after propofol-remifentanil-based anaesthesia. Eur J Anaesthesiol 2010;27:41-6.

28 Ortiz MP, Godoy MCdeM, Schlosser RS, et al. Effect of endovenous lidocaine on analgesia and serum cytokines: double-blinded and randomized trial. J Clin Anesth 2016;35:70-7. 